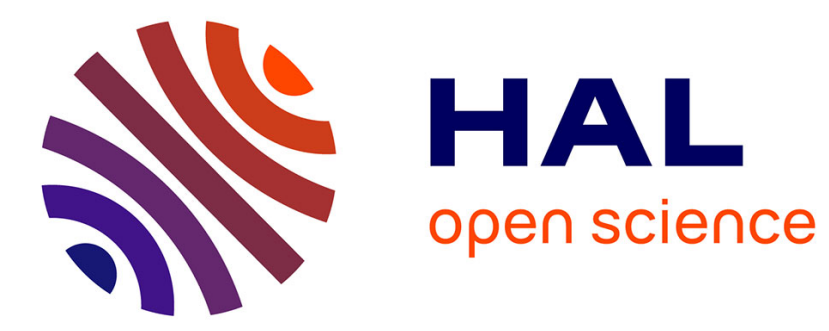

\title{
An X-ray study of brine swollen lyotropic lamellar phases
}

P. Bassereau, J. Marignan, G. Porte

\section{To cite this version:}

P. Bassereau, J. Marignan, G. Porte. An X-ray study of brine swollen lyotropic lamellar phases. Journal de Physique, 1987, 48 (4), pp.673-678. 10.1051/jphys:01987004804067300 . jpa-00210484

\section{HAL Id: jpa-00210484 https://hal.science/jpa-00210484}

Submitted on 1 Jan 1987

HAL is a multi-disciplinary open access archive for the deposit and dissemination of scientific research documents, whether they are published or not. The documents may come from teaching and research institutions in France or abroad, or from public or private research centers.
L'archive ouverte pluridisciplinaire HAL, est destinée au dépôt et à la diffusion de documents scientifiques de niveau recherche, publiés ou non, émanant des établissements d'enseignement et de recherche français ou étrangers, des laboratoires publics ou privés. 
Classification

Physics Abstracts

$61.30 \mathrm{E}-61.10 \mathrm{D}-82.70$

\title{
An X-ray study of brine swollen lyotropic lamellar phases
}

\author{
P. Bassereau, J. Marignan and G. Porte \\ Groupe de Dynamique des Phases Condensées (CNRS-UA233) \\ and GRECO Microemulsions, USTL, Place E. Bataillon, 34060 Montpellier Cedex, France
}

(Reçu le 10 novembre 1986, accepté le 5 décembre 1986)

\begin{abstract}
Résumé. - Les phases lamellaires de deux systèmes quasi ternaires (tensioactif/alcool/eau salée) sont étudiées en diffusion des rayons X. Dans les deux cas la phase présente un comportement de gonflement simple en dilution par l'eau salée, et la première singularité de Bragg persiste au moins jusqu'à $150 \AA ̊$ pour la distance interlamellaire $d$. La comparaison des profils de diffusion des échantillons les plus gonflés des deux systèmes montre que les fluctuations de l'empilement lamellaire sont beaucoup plus grandes dans un cas que dans l'autre. Cette spécificité est analysée dans le cadre de la théorie classique des fluctuations dans les milieux smectiques. Elle indique que dans le cas de ces empilements gonflés à l'eau salée, l'interaction stérique de Helfrich n'est pas la seule contribution dominante dans le potentiel d'interaction effectif entre membranes adjacentes.
\end{abstract}

\begin{abstract}
The lamellar phases of two different quasi ternary systems (surfactant/alcohol/brine) are studied by X-ray scattering. In both cases the phase exhibits simple swelling behaviour when diluted with brine and the Bragg singularity persists at least up to $150 \AA$ for the average interlamellar distance $d$. The comparison of the scattering patterns of the most swollen samples of the two systems shows that the out of plane fluctuations of the lamellar stacking are much larger in one case than in the other. This specificity is analysed in the light of the current theory on fluctuations in smectic media ; it indicates that, for the present brine swollen stackings, the Helfrich steric interaction is not the only dominant contribution to the effective intermembrane interactions.
\end{abstract}

\section{Introduction.}

Many lyotropic systems involving surfactants give rise to birefringent lamellar phases. They consist of a regular stacking of alternate hydrophilic and hydrophobic layers and in this respect they exhibit a smectic periodicity.

They differ from thermotropic smectics, in that the average periodicity in these lyotropic phases can often be varied to a great extent by appropriate adjustment of the composition of the mixture. There are many examples in the literature where an initial lamellar phase with a moderate pitch can incorporate very large amounts of added water [1-5] or respectively oil [6-9]. The resulting mixture then consists of thin hydrophobic (or conversely hydrophilic) layers separated by very thick water (or conversely oil) layers.

Especially interesting are the situations where the average interlamellar distance $d$ is made so much larger than the distances beyond which the interactions involved in colloid dispersions vanish $\left(\sim 10^{2} \AA\right)$ : attractive Van der Waals forces which quickly decay as $d^{-4}$, screened electrostatic repulsion and hydration forces. Then, it is expected $[8,11]$ that the stability of the phase is mainly determined by the long range steric interaction which has been introduced and evaluated by Helfrich [12]. It arises from the hindered bending fluctuations of the adjacent lamellae. Helfrich has calculated its free energy per unit area of membrane which scales as $d^{-2}$.

The experimental evidence of long range orientational order (optical birefringence) and of quasi long range translational order (cusp like [13] Bragg singularity in X-ray scattering) in a sufficiently swollen lamellar phase should provide a direct proof of the long range character of this interaction. Such an experimental investigation has been performed by Safinyia et al. [11] for an oil swollen lamellar phase and the quantitative fits of their X-ray scattering data indicate that the Helfrich steric interaction is in fact dominant in this case.

We herein report an X-ray scattering investigation of the lamellar phases of two brine swollen ternary systems (surfactant/alcohol/brine). The individual lamellae are indeed charged in this case, but the electrostatic repulsions are strongly screened by the high concentration of the free ions in the swelling aqueous fluid. Such systems are thus adequate for our purpose. On the other hand, the main advantage 
of these brine swollen lamellar phases is that they exhibit a simple swelling behaviour upon dilution : extra addition of brine simply push apart the adjacent hydrophobic bilayers which keep a constant thickness, chemical composition and thus mechanical rigidity upon dilution.

Two different systems were studied - namely cetypyridinium chloride $(\mathrm{CPCl}) /$ hexanol/brine $(0.2 \mathrm{M} \mathrm{NaCl})$ and sodium octylbenzene sulfonate (OBS)/pentanol/brine $(0.5 \mathrm{M} \mathrm{NaCl})-$ in order to investigate eventual specific behaviours related to the different chemical nature of the multilayered stackings.

\section{Experiments.}

The brine rich corners of the phase diagram of the two investigated systems are represented in figures 1 and 2. In both cases the lamellar phase appears stable for the proper values of the alcohol to surfactant ratio up to very high degrees of dilution : up to $\phi_{\mathrm{w}}=0.98$ at least where $\phi_{\mathrm{w}}$ is the volume fraction of the brine in the sample.

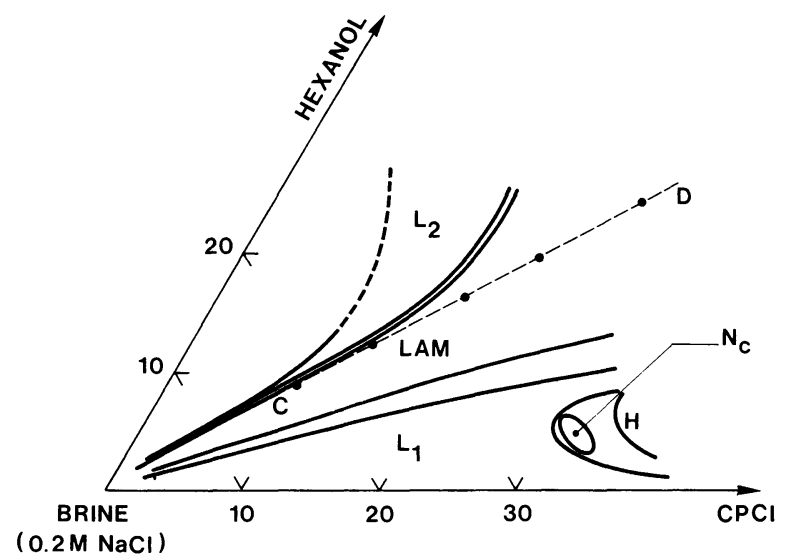

Fig. 1. - Brine rich side of the phase diagram of the system $\mathrm{CPCl} / \mathrm{Hexanol} /$ brine $(0.2 \mathrm{M} \mathrm{NaCl})$.

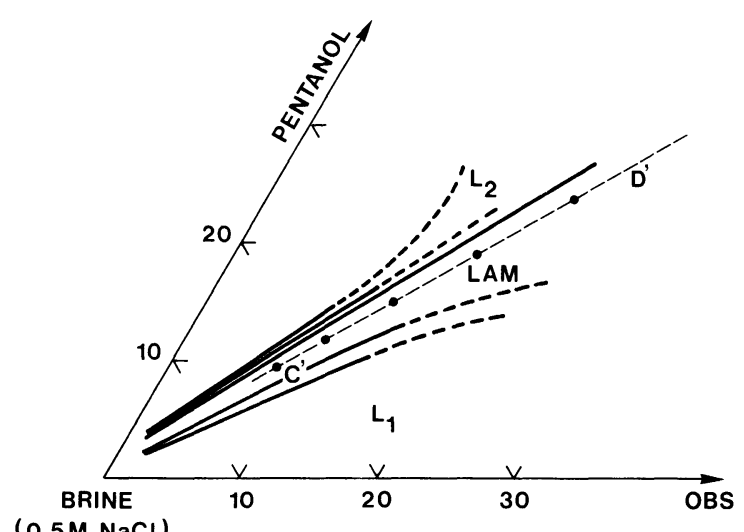

(0.5 M NaCl)

Fig. 2. - Brine rich side of the phase diagram of the system OBS/pentanol/brine $(0.5 \mathrm{M} \mathrm{NaCl})$.
Over its entire range of existence the phase remains clearly birefringent at rest. The long range orientational order thus persists up to high degrees of swelling where the interlamellar distance is expected to be of the order of $10^{3} \AA$ or more.

As discussed at length in [4], the radial geometry of the diagram in the dilute regime $\left(\phi_{w}>0.75\right)$ indicates that any straight line arising from the pure brine corner approximately corresponds to a true dilution line (along which the composition of the bilayers remain constant). The swelling behaviour of the lamellar phase of the two systems is here studied along line $\mathrm{CD}$ and $\mathrm{C}^{\prime} \mathrm{D}^{\prime}$ in figures 1 and 2, for samples ranging from $\phi_{\mathrm{w}}=0.5$ to $\phi_{\mathrm{w}}=0.83$. The samples consist of cylindrical Lindemann capillary tubes (diameter $1 \mathrm{~mm}$ ) filled with the investigated mixture. The capillary walls are thoroughly cleaned (sulfochromic acid) in order to obtain the cylindrically symetrical alignment described in figure 3 . The excellent quality of the alignment is sensitively checked by polarized light microscopy (Fig. 4).

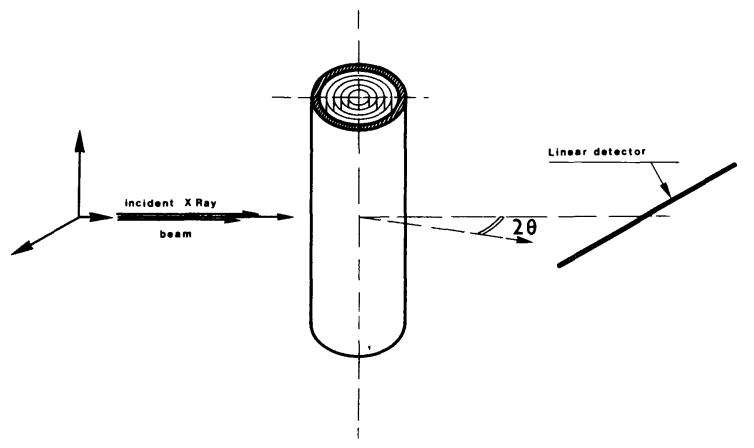

Fig. 3. - Cylindrically symetric configuration of the lamellae in the capillary tube and scattering geometry.

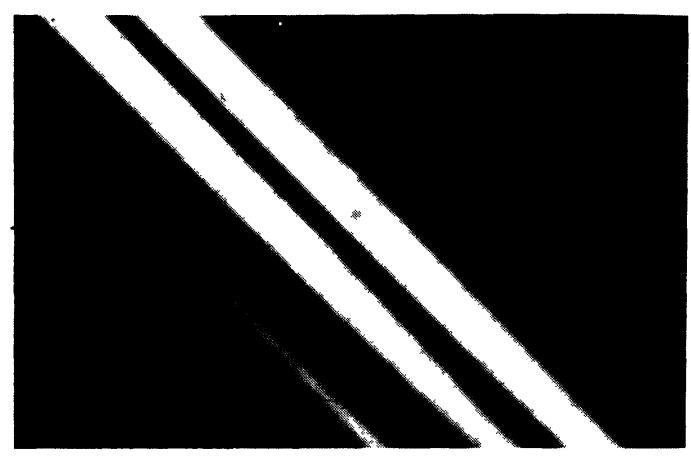

Fig. 4. - One typical sample as observed between horizontal and vertical crossed nicols.

The X-rays scattering set up of our laboratory usually works in linear collimation. Here, the incident and diffracted beams are diaphragmed in order to simulate properly a punctual configuration. Complementary data (micrographs) have been obtained 
on line $\mathrm{D}_{16}$ at the synchrotron light source in LURE. The collimation in truly punctual in this case.

Figures 5 and 6 gives the $\mathrm{X}$-rays scattering profiles obtained with the standard SAXS set up of our laboratory [10]. The axis of the capillaries is normal to the linear detector so that a substantial part of the scattering volume is illuminated according to the Bragg condition (Fig. 3).

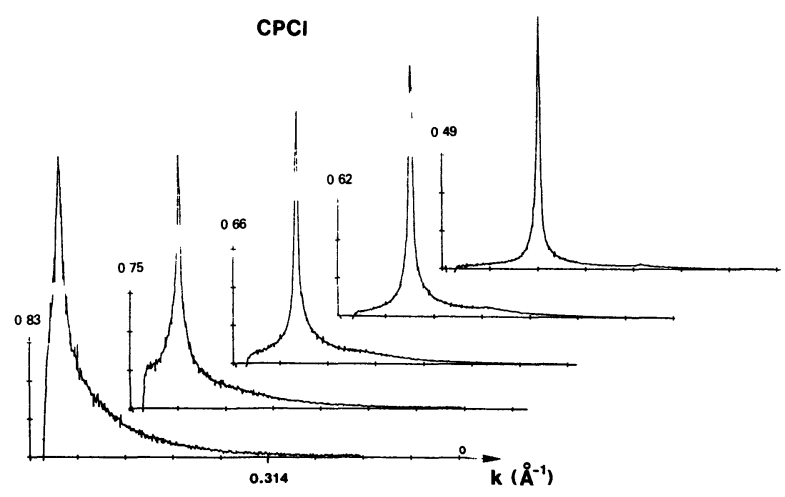

Fig. 5. - Evolution of the scattering profile as function of the dilution $\phi_{w}$ for the $\mathrm{CPCl}$ dilution line CD.

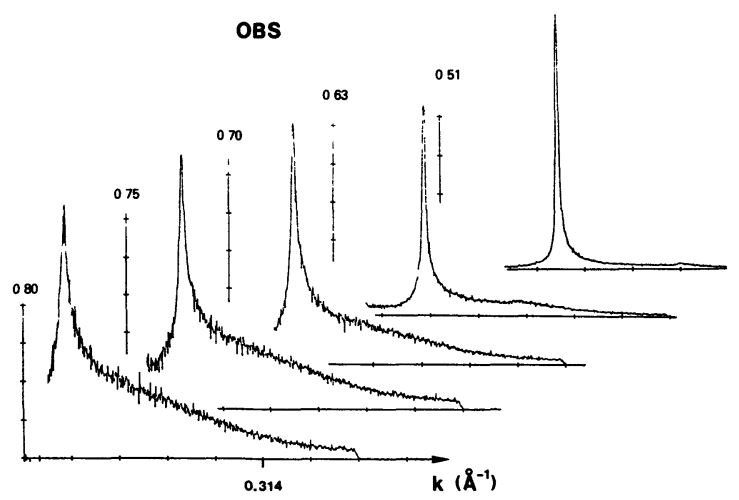

Fig. 6. - Evolution of the scattering profile as function of the dilution $\phi_{\mathrm{w}}$ for the OBS dilution line $\mathrm{C}^{\prime} \mathrm{D}^{\prime}$.

For both systems, the scattering patterns of the two most concentrated samples exhibit the two first Bragg singularities. Their relative positions on the transfer wave vector axis are in the simple ratio $1 / 2$ which is indeed suggestive of a true lamellar structure. For the other more swollen samples the second order Bragg maximum disappears. The true lamellar structure is however reliably and clearly indicated by the focal conics texture which is observed in ill oriented samples at any dilution.

In figure 7 , we have plotted the variations of the position of the first Bragg peak $(1 / d)$ as function of the degree of dilution $\phi_{w}$ for both dilution lines. They follow straight lines extrapolating down to 0 for $\phi_{\mathrm{w}}=1$. Consistently with our expectation from the radial geometry of the brine rich side of the

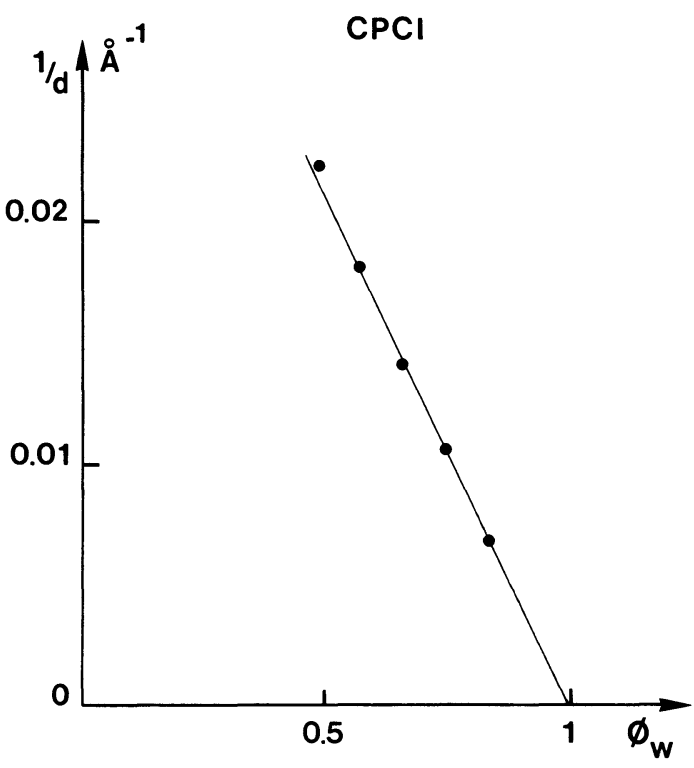

Fig. 7. - Position of the Bragg peak (expressed in $d^{-1}$ ) as function of $\phi_{\mathrm{w}}$ for the CPCl system. A similar behaviour is observed for the OBS dilution line.

phase diagrams [4], the lamellar phase exhibits a simple swelling behaviour [10] :

$$
\frac{1}{d}=\frac{1}{d_{\mathrm{a}}}\left(1-\phi_{\mathrm{w}}\right)
$$

and we can estimate the constant thickness $d_{\mathrm{a}}$ of the hydrophobic lamellae :

$$
\begin{array}{ll}
\text { CPCl : } & d_{\mathrm{a}}=26.5 \AA \\
\text { OBS : } & d_{\mathrm{a}}=20.7 \AA .
\end{array}
$$

The scattering patterns in figures 5,6 are further indicative of the persistence of the positional order upon dilution. The case of the $\mathrm{CPCl}$. system is especially clear. Up to $\phi_{w}=0.82$ the first order Bragg maximum remains very well defined. Its apex remains sharp (up to the resolution of our SAXS set up : $k \sim 3 \times 10^{-3} \AA^{-1}, \mathbf{k}$ is the wave vector) while its tail broadens widely with the dilution.

At any dilution, the intensity scattered around the Bragg position scales as $(k-2 \pi / d)^{-\nu}$ as it is clear in the $\log -\log$ plot of figure 8 . The exponent $\nu$ is a measure of the peak to tail intensity ratio. Its variation with the dilution $\phi_{w}$ is given in table I. It first decreases markedly with the dilution and then seems to level out at an asymptotic lower limit $(\nu \sim 0.6)$ for the two most swollen samples. The general pattern is thus very different from the quasi Gaussian profile with a definite width which characterizes short range positional order. It clearly indicates that, although highly swollen, the diluted lamellar stacking still presents quasi long range order [13].

In the case of the OBS system, the behaviour is qualitatively similar. However, the broadening of 


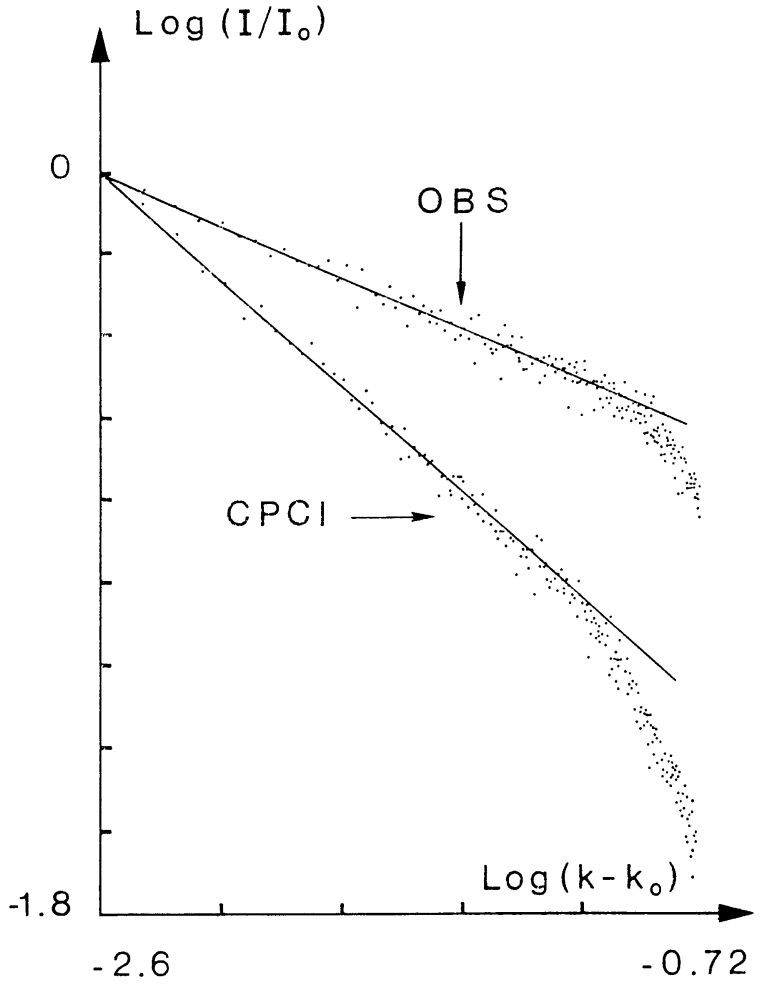

Fig. 8. - Log-log plot of the intensity as function of the deviation from the Bragg position $k_{0}$ for the swollen $\mathrm{CPCl}$ and OBS samples with $\phi_{\mathrm{w}}=0.75$.

the peak tail is much more pronounced and the peak to tail intensity ratio decreases much faster with the dilution as indicated by the evolution of the power law exponent $\nu$ in table I. Also the asymptotic lower limit of $\nu$ at high dilution is much lower $(\nu \sim 0.3)$.

These differences between the two systems are further evidenced on the micrographs (Figs. 9, 10) obtained at LURE with the same'type of oriented samples after different exposure times. For the $\mathrm{CPCl}$ system, at short time exposure (Fig. 9a) the Bragg singularity appears very well defined as two symetric small spots on the micrographs. Even after a large over exposition (Fig. 9b) of the film, most of the intensity scattered by the out of plane positional fluctuations remains localized quite close to the Bragg position. In the case of OBS sample the Bragg spot is also very well defined (Fig. 10a) although it is

Table I. - The exponent $v$ for the power law singularity as a function of the dilution $\phi_{W}$.
$\mathrm{CPCl}$
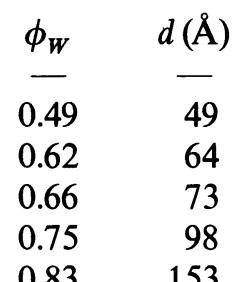

$-$

0.49

0.62

0.66

0.75

0.83

\begin{tabular}{r}
$d(\AA)$ \\
\hline 49 \\
64 \\
73 \\
98 \\
153
\end{tabular}

OBS

\begin{tabular}{cccc}
\multicolumn{1}{c}{} & $\phi_{W}$ & $d(\AA)$ & $v$ \\
- & - & - & - \\
$>0.9$ & 0.51 & 40 & $>0.9$ \\
0.9 & 0.63 & 52 & 0.61 \\
0.75 & 0.70 & 71 & 0.46 \\
0.66 & 0.75 & 84 & 0.33 \\
$0.6 \dot{4}$ & 0.80 & 112 & 0.33
\end{tabular}

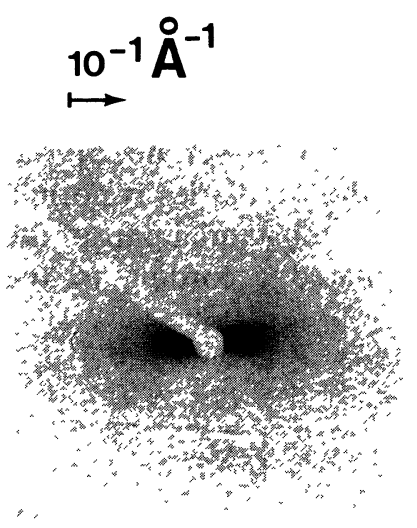

a)

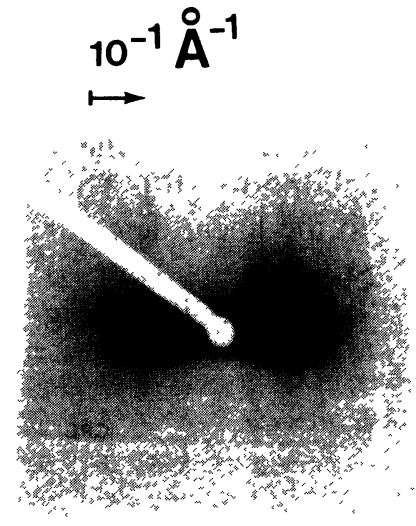

b)
Fig. 9. - 2D photographic recording of the scattering pattern of the CPCl sample with $\phi_{\mathrm{w}}=0.75$; a) short time exposure $(2.5 \mathrm{~min})$; b) long time exposure $(15 \mathrm{~min})$.

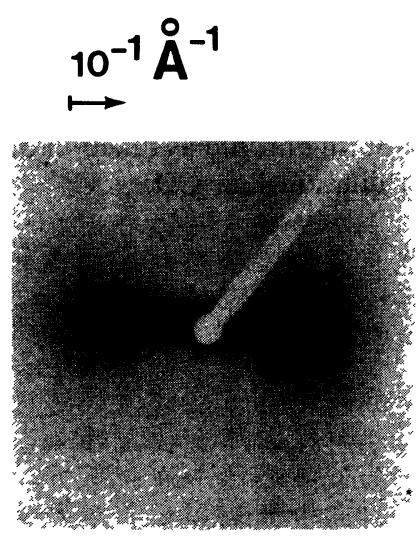

a)

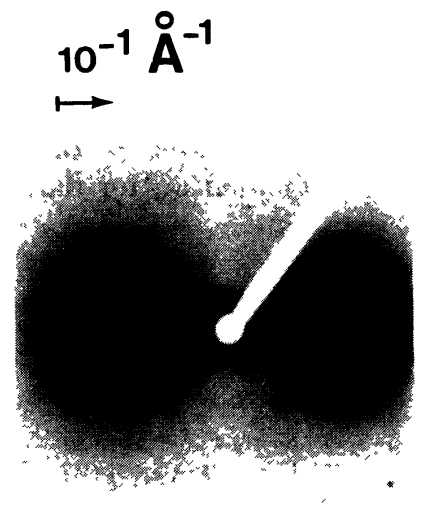

b)
Fig. 10. - 2D photographic recording of the scattering pattern of an OBS sample with $\phi_{w}=0.83$; a) short time exposure $(5 \mathrm{~min})$; b) long time exposure $(30 \mathrm{~min})$.

much less intense. But in addition we observe (Fig. 10b) a spectacular «butterfly wings » pattern which spreads over a large area of the recorded plane projection of the reciprocal space. In order to interprete these differences in terms of the interference functions only, we have modelized the electronic density distribution along the normal to one bilayer. The obtained form factor of the OBS pentanol bilayer presents a broad maximum around $k \sim 3 \times 10^{-1} \AA^{-1}$ which can partly explain the large intensity scattered at $k \gg 2 \pi / d$. On the other hand at lower $k\left(3 \times 10^{-2} \AA^{-1}<k<10^{-1} \AA^{-1}\right)$ the form factors of both systems vary smoothly with moderate slopes and thus cannot explain the observed differences around $k=2 \pi / d$. We therefore conclude that for the most swollen samples, the out of plane positional fluctuation of the bilayers are significantly larger in the case of the OBS system than in the case of $\mathrm{CPCl}$. 
Other information, related to the orientational order, is provided by the micrographs. The Bragg spots do not spread diffusively along a circle centered at the origin and having a radius equal to $2 \pi / d$. This feature indicates that for both systems the long wavelength collective orientational fluctuations, have a small average amplitude.

\section{Discussion.}

As stressed in the introduction, the usual direct interaction forces are in the present experimental conditions quickly decreasing with the distance. Two pieces of adjacent bilayers facing each other and separated by a distance larger than some $10^{2} \AA$ should be considered therefore as almost non interacting.

However, the important point in case of multilayered stackings is that these short range naked interactions are renormalized into presumably long range effective interactions by the bending fluctuations of adjacent bilayers: (although they stand on average far apart, they locally come close to each other quite frequently so that they experience from point to point the short range forces).

This expectation is well supported by the present experimental findings. The persistence of long range orientational and quasi wh' range positional orders in highly swollen lamellar samples indeed implies that some effective long range interaction couples the orientations and positions of adjacent lamellae.

The so called Helfrich steric interaction [12] arises from such a transformation of the (zero range) hard wall potential into an effective long range potential which scales as $d^{-2}$. Since then the theory has progressed $[14,16]$ and it has been argued [16] that the renormalized potentials associated with the other naked interactions (electrostatic, hydration, Van der Waals) should also involve a leading term in $d^{-2 \mu}(\mu \# 1)$ with a coefficient which depends on the rigidity $k_{\mathrm{c}}$ of the bilayers and on the detailed expressions of the bare potentials.

A question then arises naturally when considering the swelling behaviour of our systems : is the effective interaction potential mainly determined only by the Helfrich steric interaction or is it also sensitive to the renormalized contribution of the other short range forces?

We analyse this more quantitatively in the light of the current theory on fluctuations in smectic media. Within the harmonic. approximation for the elastic free energy of the lamellar stacking, the mean square $q$-mode amplitude of the positional out of plane fluctuations $u_{q}$ is :

$$
\left\langle\left|u_{q}\right|^{2}\right\rangle=k_{\mathrm{B}} T\left(\bar{B} q_{z}^{2}+\bar{K} q_{\perp}^{4}\right)^{-1},
$$

where the indices $z$ and $\perp$ respectively denote the directions normal and parallel to the smectic layers.
Here $\bar{K}$ is related to the bending rigidity modulus $k_{\mathrm{c}}$ by $\bar{K}=k_{\mathrm{c}} / d$ and $\bar{B}$ is related to the effective interaction potential per unit area for two bilayers $\Delta F / A$ by :

$$
\bar{B}=d^{2} \frac{\partial^{2}}{\partial d^{2}}\left(d^{-1} \cdot \Delta F / A\right) .
$$

One introduces for conveniency $\Lambda, x$ and $a$ :

$$
\begin{aligned}
& \Lambda=d^{-1}(\bar{K} / \bar{B})^{1 / 2} \\
& x=\frac{\pi}{2} A \frac{k_{\mathrm{B}} T}{k_{\mathrm{c}}},
\end{aligned}
$$

and $a^{-1}$ is the usual in plane $q_{\perp}$ high cut off wave vector.

The average orientational fluctuations $[7,8]$ have been calculated elsewhere :

$$
\lim _{\mathrm{r} \rightarrow \infty}\left\langle\left|\theta^{2}(\mathbf{r})\right|\right\rangle=\frac{k_{\mathrm{B}} T}{2 \pi k_{\mathrm{c}}}\left(1+\ln \left(\frac{\Lambda d^{2}}{\pi a^{2}}\right)\right),
$$

where $\theta(\mathbf{r})$ is the angle between the normals to the layers at point $r$ and point 0 . The detailed analysis of (5) shows that the first (constant) term in the bracket corresponds to the long wavelength orientational fluctuations $\left(q_{\perp} \leqslant d^{-1} \sqrt{\pi / \Lambda}\right)$. As emphasized at the end of the preceding section, we have the experimental evidence that these fluctuations are small, which implies that, for both systems, $k_{\mathrm{c}}$ is at least of the order of (or larger than) $k_{\mathrm{B}} T$.

Denoting the in-plane and out of plane coordinates of the scattering wave vector $\mathbf{k}$ by respectively $k_{\perp} d$ and $k_{z} d$, we expect $[13,17]$ for a monocrystalline oriented sample :

$S\left(k_{\perp} d, k_{z} d\right) \propto \frac{1}{\left(k_{z}-2 \pi\right)^{2-x}} \cdot F\left(\frac{\Lambda k_{\perp}^{2}}{k_{z}-2 \pi}\right)$,

around the first order Bragg harmonic, where $F(y)$ is a regular function of $y$.

For the cylindrically symmetrical alignment of our samples, $S(\mathbf{k})$ must be averaged accordingly and we have found :

$$
S_{\mathrm{cyl}}\left(0, k_{z} d\right) \propto \frac{1}{\left(k_{z}-2 \pi\right)^{1.5-x}} G\left(\Lambda k_{z}\right),
$$

where $G(y)$ is a smooth function of $y$.

Let us now assume that the pure Helfrich steric interaction is dominant in the effective interaction potential. Helfrich has calculated $\Delta F / A$ [12] :

$$
\left(\Delta F_{\mathrm{st}} / A\right)=a_{0} \frac{\left(k_{\mathrm{B}} T\right)^{2}}{k_{\mathrm{c}} d^{2}},
$$

where $a_{0}$ is a numerical constant (independent of the physical characteristics of the system) which is not precisely known but should lie in the range $0.2<a_{0}<0.9$ (see expression (67) and the few following lines in Ref. [12]). 
From (8) and using (2), (3) and (4) one immediately derives :

$$
\Lambda_{\mathrm{st}}=\left(12 a_{0}\right)^{-1 / 2} \frac{k_{\mathrm{c}}}{k_{\mathrm{B}} T},
$$

and

$$
\nu_{\text {st }}=1.5-\frac{\left(12 a_{0}\right)^{-1 / 2} \pi}{2}=\text { constant } .
$$

Since the value of $\nu_{\text {st }}$ markedly depends on the unknown exact value of $a_{0}$, a direct comparison with the measured power law exponent (Table I) is probably not very significant. More important is the fact that, whatever the precise value of $a_{0}, \nu_{\text {st }}$ as expressed in (10) is a true constant and does not depend on the physical characteristics of the system $\left(k_{\mathrm{c}}, d, T\right)$.

Thus if the steric interaction alone is the leading contribution to the effective interaction potential we should obtain similar values of $\nu$ for the most swollen samples of the two systems. We rather obtain $\nu_{\mathrm{CPCl}} \sim 0.6$ and $\nu_{\mathrm{OBS}} \sim 0.3$. The difference is far beyond the experimental inaccuracy.

We must therefore admit that, in the cases of the present brine swollen lamellar phases, the expression
(8) for the effective potential is inadequate. More precisely we guess that $(\Delta F / A)$ still scales as $d^{-2}$ at large $d$ but with a coefficient being sensitive to the short range part of the bare interaction potential and thus strongly system dependent.

At the present time, we have no quantitative description of the presumably important electrostatic contribution to the bare potential and a more detailed analysis is out of reach. Nevertheless it is probably significant that the system with the « harder» swelling behaviour $(\mathrm{CPCl} /$ hexanol/brine $0.2 \mathrm{M})$ corresponds to the brine with the smaller ionic strength and thus the larger Debye screening length.

\section{Acknowledgments.}

We gratefully acknowledge J. Charvolin for fruitful discussions and D. Sornette, D. Roux and S. Leibler for communication of preprints on their work before publication. This research has received partial financial support from a PIRSEM (CNRS) grant number No AIP2004. One of us (P. Bassereau) is supported by Direction des Recherches Etudes et Techniques (grant No 85/1407).

References

[1] Ekwall, P., in Advances in Liquid Crystals, Edited by G. M. Brown (Academic, New York) 1975.

[2] Benton, W. J., Miller, C. A., J. Phys. Chem. 87 (1983) 4981.

[3] Marbich, W., Helfrich, W., Chem. Phys. Lipids 36 (1984) 39.

[4] Porte, G., Gomati, R., El Haitamy, O., Appell, J., Marignan, J., J. Phys. Chem. 90 (1986) 5746.

[5] Hauser, M., Gains, N., Lasic, D. D., In Physics of amphiphiles; micelles, vesicles and microemulsions (North Holland) ed. De Giorgio V. and Corti M. 1985, p. 648.

[6] Roux, D., Thesis, Bordeaux (1984).

[7] Di Meglio, J. M., Dvolaitzky, M., Taupin, C., J. Phys. Chem. 89 (1985) 871.

[8] Larche, F. C., Appell, J., Porte, G., Bassereau, P., Marignan, J., Phys. Rev. Lett. 56 (1986) 1700.
[9] Larche, F. C., Marignan, J., El Quebbaj, S., J. Phys. Chem. 90 (1986) 707.

[10] Marignan, J., Delichere, A., Larche, F. C., J. Physique Lett. 44 (1983) L-609.

[11] Safinya, C. R., Roux, D., Smith, G. S., Sinha, S. K., Dimon, P., Clark, N. A., Belloce, A. M., Phys. Rev. Lett. 57 (1986) 2518.

[12] HELFRICH, W., Z. Naturforsch 33a (1978) 305.

[13] Gunther, L., IMry, Y., Lajzerowicz, J., Phys. Rev. A 22 (1980) 1733.

[14] SornetTE, D., Europhys. Lett. 2 (1986) 715.

[15] Lipowski, R., Leibler, S., Phys. Rev. Lett. 56 (1986) 2541.

[16] SORNETTE, D., Tricritical behaviour in lamellar phases, Preprint (1986).

[17] Caille, A., C.R. Heb. Séan. Acad. Sci. B 274 (1972) 891. 